\title{
Caractérisation des populations naturelles d'une légumineuse alimentaire sauvage, Senegalia macrostachya (Reichenb. ex DC. Kyal \& Boatwr) dans le secteur Nord-soudanien du Burkina Faso
}

\author{
Youssouf SAWADOGO ${ }^{1}$, Souleymane GANABA ${ }^{2}$, Elycée TINDANO $^{3 *}$ et \\ Antoine N SOME ${ }^{1}$ \\ ${ }^{1}$ Université Nazi Boni, 01 BP 1091 Bobo-Dioulasso 01, Burkina Faso. \\ ${ }^{2}$ Institut de l'Environnement et de Recherches Agricoles, \\ Département Environnement et Forêts, BP 4047 Ouagadougou 03, Burkina Faso. \\ ${ }^{3}$ Université Ouaga I Pr Joseph Ki-Zerbo, BP: 7021 Ouagadougou 03, Burkina Faso. \\ *Auteur correspondant ; E-mail : elisetindano82@yahoo.fr
}

\section{REMERCIEMENTS}

Nous remercions le Fonds National de la Recherche et de l'Innovation pour le Développement (FONRID) du Burkina Faso, pour le financement de la présente étude.

\section{RESUME}

Au Burkina Faso, Senegalia macrostachya (Reichenb. ex DC. Kyal \& Boatwr) est une légumineuse sauvage dont les graines sont consommées. Elles sont cuites et consommées comme le niébé pendant les cérémonies. La présente étude sur cette espèce a été menée dans les provinces du Gourma, du Kourwéogo et du Sourou. Elle a pour objectif d'évaluer l'état des populations de l'espèce dans les jachères et les savanes. Pour ce faire, un inventaire floristique et des mesures dendrométriques des formations végétales des sites d'étude ont été réalisés. Les mesures dendrométriques ont concerné les individus dont le diamètre à la base est supérieur ou égal à $3 \mathrm{~cm}$. Au total, dix-huit (18) placettes permanentes circulaires, de $5000 \mathrm{~m}^{2}$ (1/2 ha) de superficie, ont été réparties sur les trois $(03)$ sites. L'inventaire a permis de recenser 91 espèces ligneuses réparties en 55 genres et 28 familles. Les familles, les mieux représentées en termes d'espèces sont les Combretaceae et les FabaceaeMimosoïdeae. L'espèce Senegalia macrostachya présente un potentiel important dans les jachères (141 \pm 78 individus/ha, $187 \pm 127$ individus/ha, $155 \pm 96$ individus/ha) respectivement dans les provinces du Gourma, du Kourweogo et du Sourou ainsi que dans les savanes $(315 \pm 169$ individus/ha, $191 \pm 87$ individus/ha, $103 \pm 22$ individus/ha) respectivement dans les provinces du Gourma, du Kourweogo et du Sourou avec des faibles taux de régénération dans les jachères $(21,80 \% ; 16,01 \% ; 27,90 \%)$ et dans les savanes $(37,08 \% ; 25,09 \% ; 25,81 \%)$ respectivement dans les provinces du Gourma, du Kourweogo et du Sourou. Ces résultats peuvent servir à la conservation et à la valorisation de l'espèce. Cependant, elle est menacée par les faibles taux de régénération qu'elle présente.

(C) 2017 International Formulae Group. All rights reserved.

Mots clés: Senegalia macrostachya, légumineuse alimentaire, inventaire floristique, jachère, savane, Burkina Faso. 


\title{
Characterization of the natural populations of a wild food legume, Senegalia macrostachya (Reichenb.e. DC, Kyal \& Boatwr) in the Northern Sudanian of Burkina Faso
}

\begin{abstract}
Senegalia macrostachya (Reichenb. ex DC. Kyal \& Boatwr) is a leguminous wild plant of Burkina Faso whose seeds are consumed. They are cooked and consumed as cowpea during ceremonies. The current study concerning this species was realized in the provinces of Gourma, Kourwéogo and Sourou. It aimed at evaluating to evaluate the biological potential of the plant in the various types of vegetable formations. With this intention, a floristic inventory of the sites of study was carried out. Eigten (18) permanent small squares were distributed in three sites. The small squares are of circular form with a radius of $39.89 \mathrm{~m}$ and a surface of $1 / 2$ ha. From the inventory, 91 woody species divided into 55 kinds and 28 families were obtained. The families, best represented in terms of species are Combretaceae and Fabaceae-Mimosoïdeae. The species Senegalia macrostachya showed an important potential in the fallow $(141 \pm 78$ individus/ha, $187 \pm 127$ individus/ha, $155 \pm 96$ individus/ha) in order in the provinces of Gourma, Kourwéogo and Sourou and savannas (315 \pm 169 individus/ha, $191 \pm 87$ individus/ha, $103 \pm 22$ individus/ha) in orders in the provinces of Gourma, Kourwéogo and Sourou but these regeneration rate are weaker in the fallow $(21,80 \% ; 16,01 \%$; $27,90 \%)$ than the savannas $(37,08 \% ; 25,09 \% ; 25,81 \%)$ in order in the provinces of Gourma, Kourwéogo and Sourou. These results could be used for the conservation and valuing of the species. However, it is threatened by the poor regeneration rate.

(C) 2017 International Formulae Group. All rights reserved.
\end{abstract}

Keywords: Senegalia macrostachya, food leguminous plant, floristic inventory, fallow, savanna, Burkina Faso

\section{INTRODUCTION}

Compte tenu de l'extrême pauvreté qui sévit dans les zones rurales, les populations locales qui y vivent dépendent largement des ressources naturelles renouvelables pour la satisfaction de leurs besoins. Elles les exploitent comme complément alimentaire et comme source de revenus par la vente du bois ou des produits forestiers non ligneux (pfnl) (Traoré et al., 2011 ; Thiombiano et al., 2012). Cependant, à l'instar des autres espèces, les espèces ligneuses alimentaires subissent une dégradation à travers la dégradation des écosystèmes, de l'érosion de la diversité biologique et à leur utilisation non rationnelle (Mouamfon et al., 2015). Selon Eyog Matig et al. (2002) et Ganaba (2011), cette dégradation est due aux défrichements anarchiques des forêts pour l'agriculture, aux excès de prélèvement des organes comestibles, à la croissance démographique, à l'utilisation intensive des ressources naturelles et à l'expansion de l'activité minière. A cela s'ajoute le vieillissement des peuplements en raison de l'insuffisance de la régénération et de la concurrence des autres espèces envahissantes moins exigeantes face à des conditions écologiques de plus en plus défavorables du milieu.

Parmi les ligneux pourvoyeurs de pfnl au Burkina Faso, nous pouvons citer Senegalia macrostachya (zamne en langue national san) qui est une espèce arbustive répartie dans toute la zone soudano-sahélienne (Arbonnier, 2000; Wittig et al., 2004) avec d'énormes potentialités aussi sur le plan alimentaire que médicinal. Les graines de Senegalia macrostachya sont bouillies et consommées au cours des fêtes et des cérémonies dans les grandes villes du pays 
(Eyog Matig et al., 2002). Toutes les parties de l'espèce sont utilisées dans la médecine traditionnelle. Les feuilles, les jeunes rameaux et l'écorce sont utilisés pour soigner les maux d'estomac et de dents ou comme vermifuges. En cas de morsures de serpent, la consommation en grande quantité des feuilles de Senegalia macrostachya empêcherait la diffusion du venin dans le sang (Arbonnier, 2000). Depuis un certain temps, l'exploitation des fruits de cette espèce est devenue proliférant du fait que la vente de ces graines présente une activité générant des revenus. Ceci a entraîné une surexploitation des fruits de l'espèce qui connaît une forte régression de ses populations du fait de la pression anthropique et animale, des feux de brousse etc. (Eyog Matig et al., 2002). Vu la pression que subit l'espèce, qu'en est-il de son état de peuplement dans les milieux qu'elle colonise?

C'est dans ce sens que nous caractériserons l'état actuel du potentiel disponible de Senegalia macrostachya des différents sites d'étude à travers un inventaire floristique et des mesures dendrométriques. L'objectif de cette étude est d'évaluer le potentiel disponible de cette espèce dans les différents types de formations végétales. L'hypothèse de recherche sous-tendant cette étude envisage que Senegalia macrostachya possède un potentiel important dans les différents types de formations végétales et que son état de peuplement est stable et équilibré. Une bonne connaissance du potentiel de l'espèce pourrait permettre d'organiser l'exploitation des différentes parties de l'arbre qui procure du bien être aux populations rurales afin de pérenniser l'espèce. Une domestication de l'espèce pourrait être envisagée.

\section{METHODES D'ETUDE}

\section{Milieu d'étude}

L'étude a été menée suivant un gradient latitudinal Nord-Sud dans trois provinces (Sourou, Kourwéogo et Gourma) du secteur Nord-soudanien (Figure 1). Le choix du secteur nord-soudanien a été influencé du fait qu'il soit la zone de prédilection de l'espèce Senegalia machrostachya. Il est situé entre les latitudes $13^{\circ} \mathrm{N}$ et $11^{\circ} 30^{\prime} \mathrm{N}$. Il est caractérisé par une pluviométrie annuelle qui varie entre 600 et $900 \mathrm{~mm}$ et une saison sèche de Novembre à Mai (Dipama, 2010). Selon les données de la Direction de la Météorologie Nationale, les données pluviométriques couvrant la période 1992 à 2011 situent les moyennes annuelles de pluviosité des sites d'étude à $861,85 \mathrm{~mm}, 679,98 \mathrm{~mm}$ et 703,35 $\mathrm{mm}$ respectivement dans les provinces du Gourma, du Kourwéogo et du Sourou. On note une végétation de savanes arbustive et arborée, avec la présence de forêts claires et de forêts galeries le long des cours d'eau permanents ou temporaires (MECV, 2004). La végétation se dégrade rapidement du fait essentiellement du surpâturage, de la coupe abusive du bois, des feux de brousse et à la péjoration climatique. Les savanes sont dominées par de gros arbres de 10 à $20 \mathrm{~m}$ de hauteur appartenant aux espèces protégées (MECV, 2004).

\section{Echantillonnage}

L'échantillonnage adopté est un sondage stratifié (Bognounou, 2009) dont le critère de stratification était la présence de l'espèce Senegalia macrostachya. Le nombre de placettes a été de 18 au total dont 6 par site (3 sites) et 3 par type de formation végétale (jachère et savane) dans chaque site. Les placettes sont de forme circulaire avec un rayon de $39,89 \mathrm{~m}$ et d'une superficie de 5000 
$\mathrm{m}^{2}$ (1/2 ha). Des placettes d'une telle taille ont été préférées pour que la placette puisse contenir un échantillon d'au moins trente (30) pieds de Senegalia macrostachya. Selon Soloviev et al. (2009), la présence effective d'un peuplement naturel ou artificiel n'est confirmée que si le groupe de sujets répertorié contient au minimum 30 arbres relativement groupés.

\section{Collecte des données}

L'inventaire floristique a concerné la strate ligneuse dans le but de présenter la formation ligneuse $\mathrm{du}$ point de vue composition floristique. Au niveau de chaque placette, un comptage exhaustif des ligneux a été réalisé. Pour chaque individu adulte, les paramètres suivants ont été relevés: les coordonnées GPS, le nombre de tiges, le diamètre à $30 \mathrm{~cm}$ du sol. La mesure a été faite à $30 \mathrm{~cm} \mathrm{du}$ sol pour tenir compte des ramifications et de l'aspect touffu de certaines espèces (Bognounou, 2009). La hauteur totale des individus pour établir la structure verticale $\mathrm{du}$ peuplement et le diamètre du houppier, deux mesures dans deux directions orthogonales du petit et grand diamètre, ont été mesurées pour estimer le recouvrement. Pour les individus multicaules, la moyenne des diamètres a été calculée. La régénération a été énumérée et a concerné les individus de toutes les espèces dont le diamètre à la base est inférieur à $3 \mathrm{~cm}$. La vitalité des individus a été appréciée à l'aide d'une échelle à cinq (5) niveaux: 1 = individu sain, $2=$ individu malade (attaques, présence de galle etc.), $3=$ individu desséché, $4=$ branches arrachées, $5=$ pied mort. Ces mesures dendrométriques ont permis d'apprécier les populations de Senegalia macrostachya à travers leur structure, leur densité et leur dynamisme.

\section{Analyse et traitement des données}

Une liste floristique comportant le nom, le genre et la famille de chaque espèce a permis d'apprécier la richesse floristique des sites d'études. Le logiciel JMP a été utilisé pour les analyses de variance (ANOVA) au seuil de 5\%.

Les paramètres dendrométriques ont été calculés de la manière suivante:

- la densité des ligneux à l'hectare (D) exprimée en nombre de pieds par hectare $\mathbf{D}=\left[(\mathbf{N} / \mathbf{S})^{*} \mathbf{1 0 0}\right]$, avec $\mathrm{D}=$ densité, $\mathrm{N}=$ nombre d'individus ou de pieds de l'espèce, $\mathrm{S}$ $=$ surface totale,

- la surface terrière (ST) exprimée en $\mathrm{m}^{2} / \mathrm{ha}$ $\mathbf{S T}=[(\mathbf{D B} * \pi) / 4]$, avec DB: diamètre à $30 \mathrm{~cm}$ du sol (Tindano et al., 2014).

La structure horizontale des peuplements a été établie en répartissant les individus de l'espèce par classe de diamètre à intervalle $3 \mathrm{~cm}$ ([3-6[; [6-9[; [9-12[; [12-15[et $\geq 15$ ). La structure verticale des peuplements a été établie par la répartition des individus en classes de hauteur d'intervalle $1 \mathrm{~m}$ ([0-1[; [12[; [2-3[; [3-4[et $\geq 4 \mathrm{~m}$ ). Les effectifs par classe ont permis de tracer des histogrammes de distribution qui donnent la structure de l'espèce (Tindano et al., 2011).

\section{- le taux de régénération}

La régénération a été déterminée dans chaque formation végétale par le calcul du nombre d'individus jeunes susceptibles de remplacer les pieds adultes à long terme (Ouédraogo, 2006).

Taux de régénération $=[\mathrm{Nj} /$ $(\mathrm{Nj}+\mathrm{Na})]^{* 100}$

Potentiel de renouvellement $=(\mathrm{Nj} / \mathrm{Na}) * 100$, $\mathrm{Nj}$ : nombre de jeunes pieds, Na: nombre de pieds adultes. 


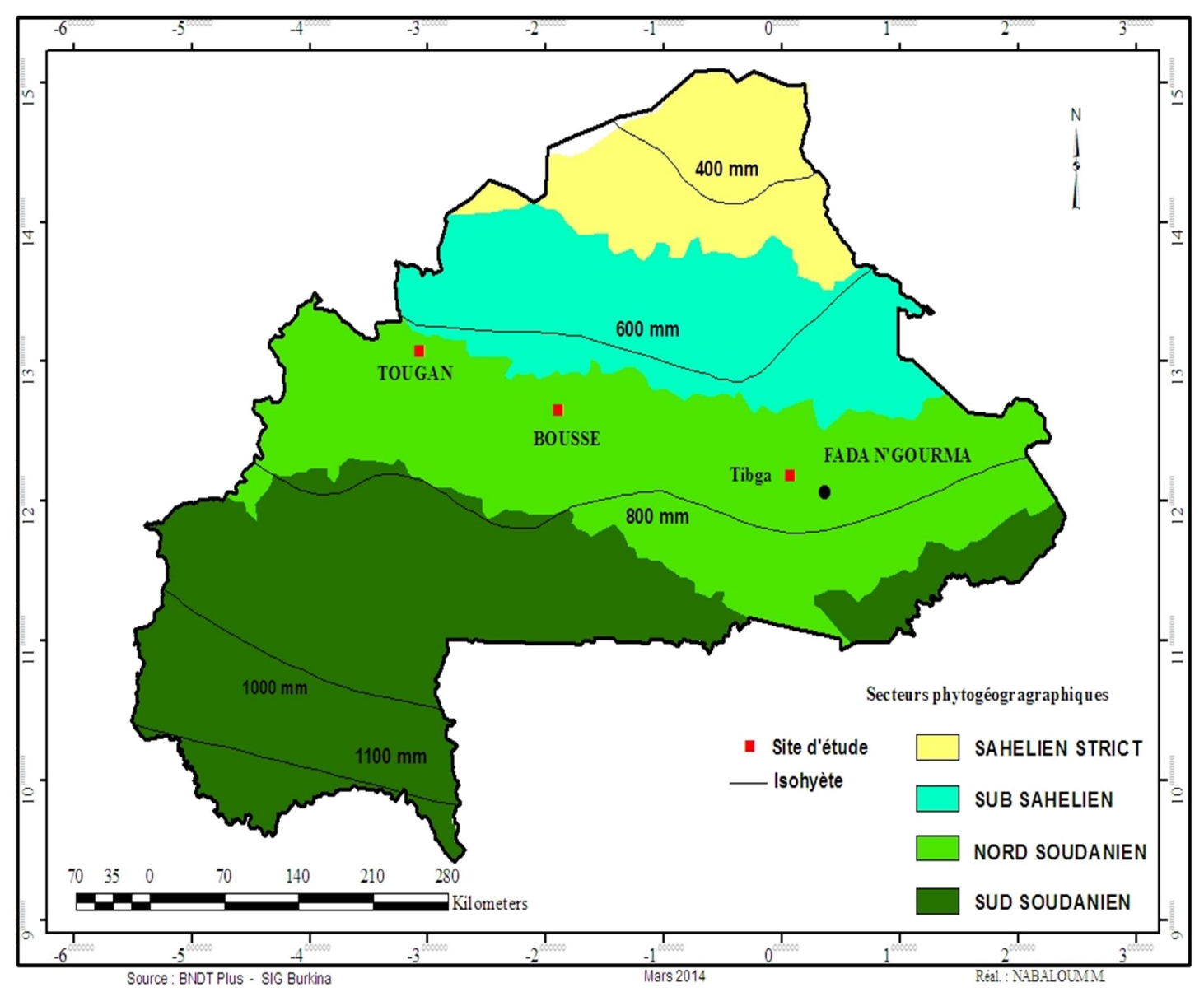

Figure 1: Carte de localisation des sites d'étude.

\section{RESULTATS}

\section{Composition floristique des sites}

L'inventaire floristique a permis de recenser au total 91 espèces ligneuses réparties en 55 genres et 28 familles. Soixante-seize (76) espèces ligneuses appartenant à 48 genres et 26 familles ont été inventoriées dans les jachères contre 72 espèces ligneuses réparties en 46 genres et 24 familles dans les savanes. Les familles les plus représentées sont les Combretaceae $(15,58 \%$ et $15,49 \%)$ et les FabaceaeMimosoïdeae $\quad(15,58 \%$ et $15,49 \%)$ respectivement dans les jachères et les savanes en termes d'espèces (Tableau 1).

\section{Caractéristiques dendrométriques de Senegalia macrostachya}

L'analyse de la variance de la densité ne montre pas de différence significative entre les jachères et les savanes $(\mathrm{P}<0,026 ; \mathrm{P}<0,13$ $\mathrm{P}<0,087)$ respectivement dans les provinces du Gourma, Kourwéogo et Sourou (Tableau 2). L'analyse de la variance de la surface terrière ne montre pas également de différence significative entre les jachères et les savanes avec des p-value supérieures à $0,05(\mathrm{P}<$ 
0,071; $\mathrm{P}<0,27 ; \mathrm{P}<0,23)$ dans les provinces du Gourma, Kourwéogo et Sourou (Tableau 2).

Dynamique des populations de Senegalia macrostachya

Structure horizontale

Les histogrammes de la structure horizontale présentent des formes en «L» avec des courbes de tendance exponentielle décroissantes (Figure 2). Une faible représentativité d'individus dans la classe de diamètre $[0-3$ [est remarquée par rapport à celle de la classe de diamètre [3-6[dans les jachères ainsi que dans les savanes. A partir de la classe de diamètre [3-6[, l'effectif des individus diminue au fur et à mesure que le diamètre augmente. Les valeurs du coefficient de détermination $\mathrm{R}^{2}$, sont $\geq 0,50\left(\mathrm{R}^{2}=0,81\right.$; $\mathrm{R}^{2}=0,80 ; \mathrm{R}^{2}=0,83$ dans les jachères et $\mathrm{R}^{2}=$ 0,$83 ; \mathrm{R}^{2}=0,65 ; \mathrm{R}^{2}=0,72$ dans les savanes) respectivement dans les provinces du Gourma, Kourwéogo et Sourou (Figure 2).

\section{Structure verticale}

Pour la structure verticale de Senegalia macrostachya, les histogrammes présentent des allures en «L» avec des courbes de tendance exponentielle décroissantes dans les jachères et les savanes dans les provinces du Kourwéogo et du Gourma (Figure 3). Les valeurs du coefficient de détermination $\mathrm{R}^{2}$ sont élevées $\geq 0,50\left(\mathrm{R}^{2}=0,56 ; \mathrm{R}^{2}=0,56\right.$ dans les jachères et $\mathrm{R}^{2}=0,82 ; \mathrm{R}^{2}=0,68$ dans les savanes) respectivement dans les provinces du Gourma et Kourwéogo (Figure 3). Pour la structure verticale de l'espèce de la province $\mathrm{du}$ Sourou, les histogrammes présentent des allures en cloche avec des courbes de tendance exponentielle légèrement décroissantes dans les jachères et dans les savanes. De la classe [0-1[à la classe de hauteur [1-2[, il y'a une diminution importante $\mathrm{du}$ nombre d'individus. Les individus sont plus nombreux dans les classes de hauteur [2-3[et à partir de cette classe le nombre d'individus diminue au fur et à mesure que la hauteur augmente.

\section{Régénération de Senegalia macrostachya}

Le nombre d'individus juvéniles de Senegalia macrostachya est faible par rapport au nombre des adultes dans les jachères et dans les savanes. Ce qui donne des taux de régénération $<50 \% \quad(21,80 \% ; 16,01 \%$; $27,90 \%$ dans les jachères et $37,08 \% ; 25,09 \%$; $25,81 \%$ dans les savanes) respectivement dans les provinces du Gourma, Kourwéogo et Sourou (Figure 4).

\section{Vitalité de Senegalia macrostachya}

Les individus de Senegalia macrostachya recensés dans les jachères présentent, dans l'ensemble, un bon état de santé avec respectivement $67,38 \%$ et $89,82 \%$ d'individus sains pour les provinces du Kourwéogo et du Sourou. Par contre, dans la province du Gourma, le taux d'individus sains est de 44,51\%. Dans les savanes, les individus de Senegalia macrostachya présentent également un bon état de santé avec respectivement $70,03 \%, 90,79 \%$ et $88,79 \%$ d'individus pour les provinces du Gourma, du Kourwéogo et du Sourou. Pour les pieds de Senegalia macrostachya, les différentes mutilations rencontrées sont principalement les coupes dans les jachères et dans les savanes surtout au niveau de la province du Gourma avec des taux de $46,34 \%$ et $15,15 \%$ respectivement dans les jachères et dans les savanes. 
Tableau 1: Représentativité des différentes familles des formations végétales dans les jachères et dans les savanes.

\begin{tabular}{|c|c|c|}
\hline Familles & Savane (nombre d'espèces) & Jachère (nombre d'espèces) \\
\hline Combretacées & $11(15,49 \%)$ & $12(15,58 \%)$ \\
\hline Fabacées-mimosoïdées & $11(15,49 \%)$ & $12(15,58 \%$ \\
\hline Rubiacées & $7(9,86 \%)$ & $7(9,09 \%)$ \\
\hline Capparacées & $6(8,45 \%)$ & $2(2,6 \%)$ \\
\hline Anacardiacées & $5(7,04 \%)$ & $6(7,79 \%)$ \\
\hline Fabacées-césalpinoïdées & $5(7,04 \%)$ & $6(7,79 \%)$ \\
\hline Fabacées-faboïdées & $4(5,63 \%)$ & $4(5,19 \%)$ \\
\hline Tiliacées & $4(5,63 \%)$ & $3(3,9 \%)$ \\
\hline Apocynacées & $2(2,82 \%)$ & $2(2,6 \%)$ \\
\hline Burséracées & $2(2,82 \%)$ & $1(1,3 \%)$ \\
\hline Euphorbiacées & $2(2,82 \%)$ & $4(5,19 \%)$ \\
\hline Annonacées & $1(1,41 \%)$ & $1(1,3 \%)$ \\
\hline Balanitacées & $1(1,41 \%)$ & $1(1,3 \%)$ \\
\hline Bignoniacées & $1(1,41 \%)$ & $1(1,3 \%)$ \\
\hline Bombacacées & $1(1,41 \%)$ & $2(2,6 \%)$ \\
\hline Célastracées & $1(1,41 \%)$ & $1(1,3 \%)$ \\
\hline Ebénacées & $1(1,41 \%)$ & $1(1,3 \%)$ \\
\hline Loganiacées & $1(1,41 \%)$ & 0 \\
\hline Olacacées & $1(1,41 \%)$ & $1(1,3 \%)$ \\
\hline Polygalacées & $1(1,41 \%)$ & $1(1,3 \%)$ \\
\hline Rhamnacées & $1(1,41 \%)$ & $1(1,3 \%)$ \\
\hline Sapotacées & $1(1,41 \%)$ & $1(1,3 \%)$ \\
\hline Sterculiacées & $1(1,41 \%)$ & $1(1,3 \%)$ \\
\hline Asclépiadacées & 0 & $2(2,6 \%)$ \\
\hline Lamiacées & 0 & $1(1,3 \%)$ \\
\hline Liliacées & 0 & $2(2,6 \%)$ \\
\hline Meliacées & 0 & $1(1,3 \%)$ \\
\hline
\end{tabular}


Tableau 2: Densité et Surface terrière de Senegalia macrostachya des formations végétales des sites.

\begin{tabular}{lcccc}
\hline Sites & \multicolumn{2}{c}{ Densité $/$ ha } & \multicolumn{2}{c}{ Surface terrière $\mathbf{( c m}^{\mathbf{2}} \mathbf{~}$} \\
\cline { 2 - 5 } & Jachère & Savane & Jachère & Savane \\
\hline Gourma & $141 \pm 516 \mathrm{a}$ & $315 \pm 169 \mathrm{a}$ & $26,84 \pm 33,42 \mathrm{a}$ & $22,31 \pm 17,96 \mathrm{a}$ \\
Kourwéogo & $187 \pm 127 \mathrm{a}$ & $191 \pm 87 \mathrm{a}$ & $17,67 \pm 21,29 \mathrm{a}$ & $19,53 \pm 13,47 \mathrm{a}$ \\
Sourou & $155 \pm 96 \mathrm{a}$ & $103 \pm 22 \mathrm{a}$ & $26,54 \pm 26,13 \mathrm{a}$ & $30,46 \pm 27,61 \mathrm{a}$ \\
\hline
\end{tabular}

Les lignes non connectées par la même lettre sont significativement différentes au seuil de $5 \%$.
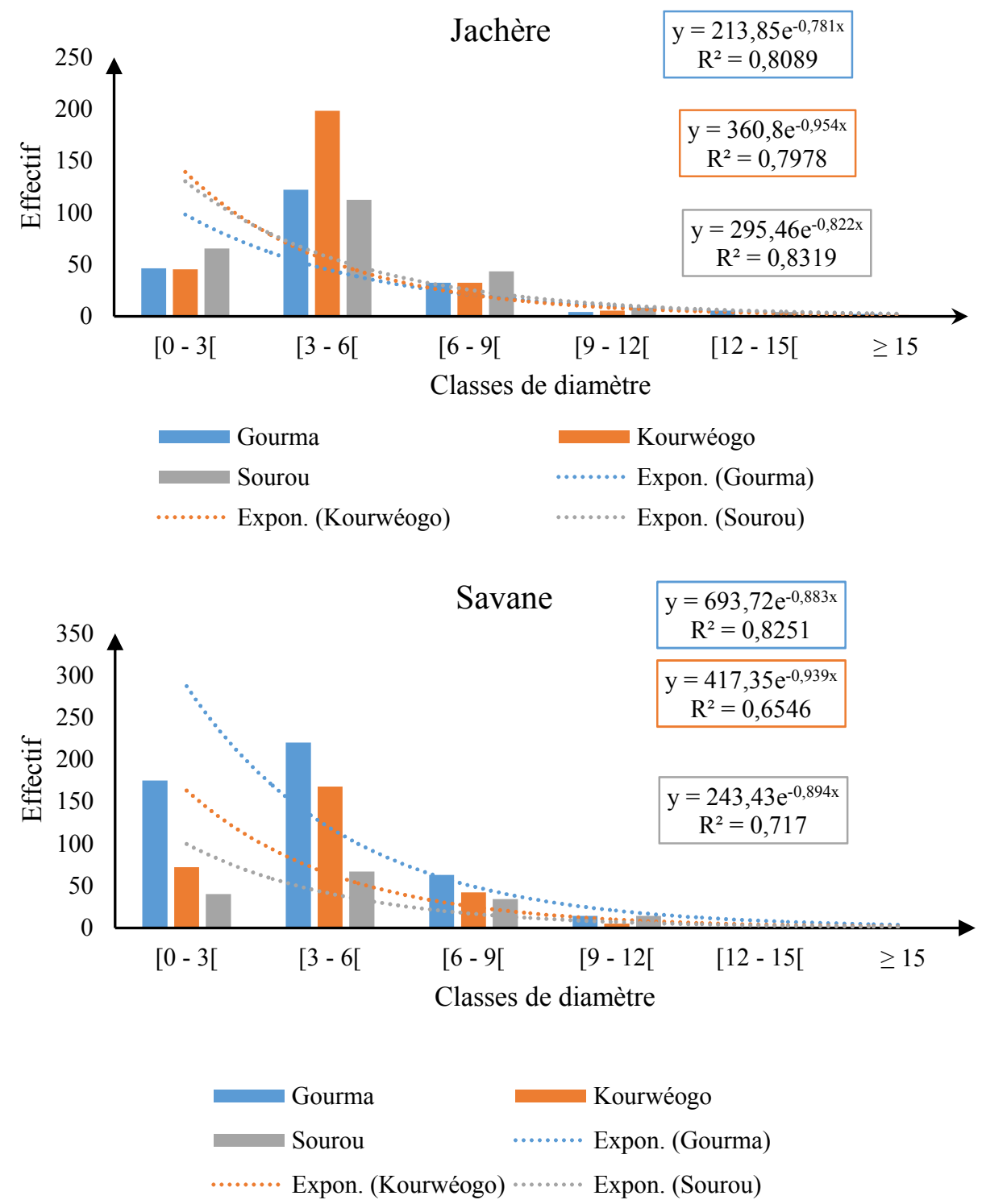

Figure 2: Structure horizontale de Senegalia macrostachya dans les différents sites. 

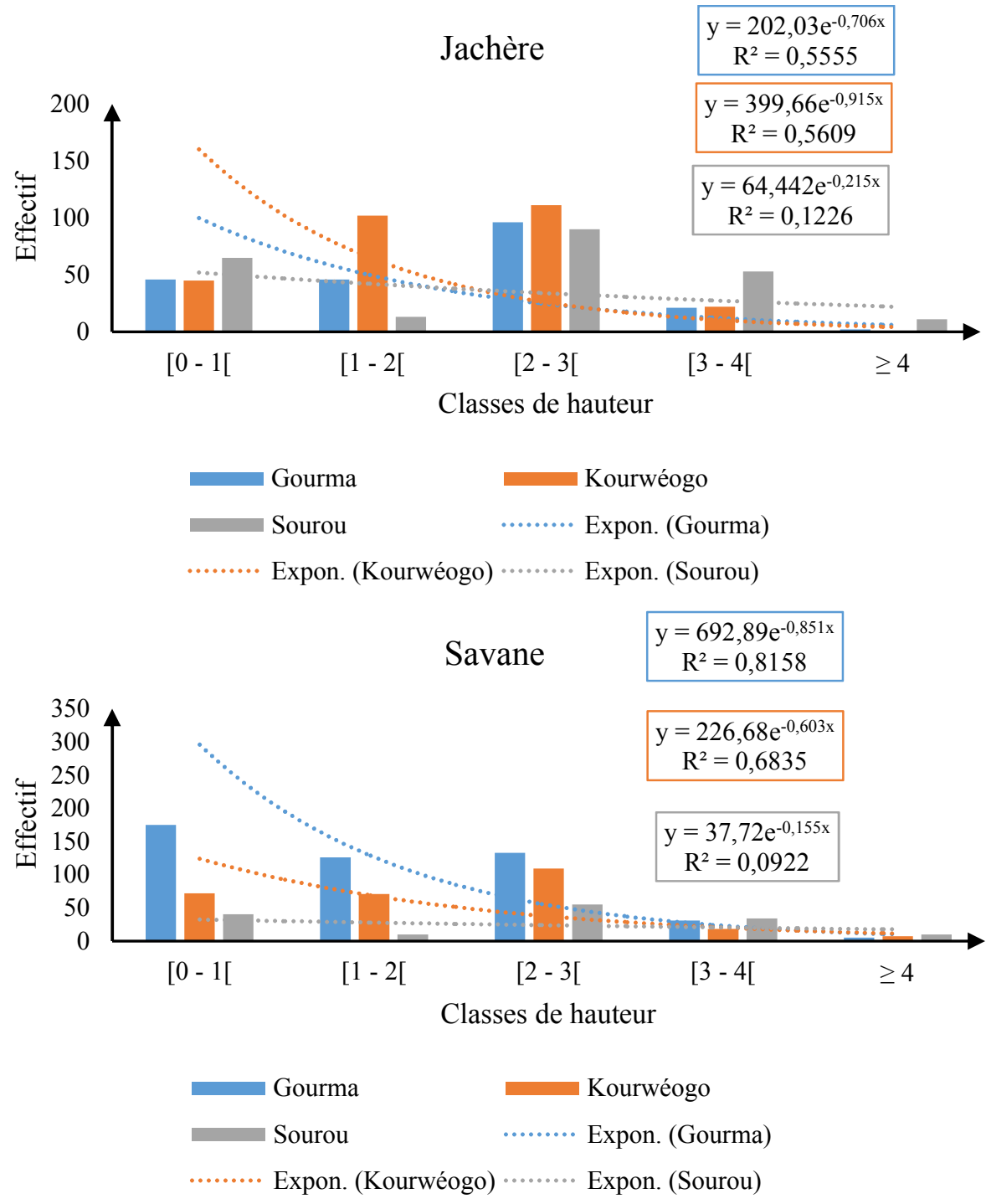

Figure 3: Structure verticale de Senegalia macrostachya dans les différents sites.

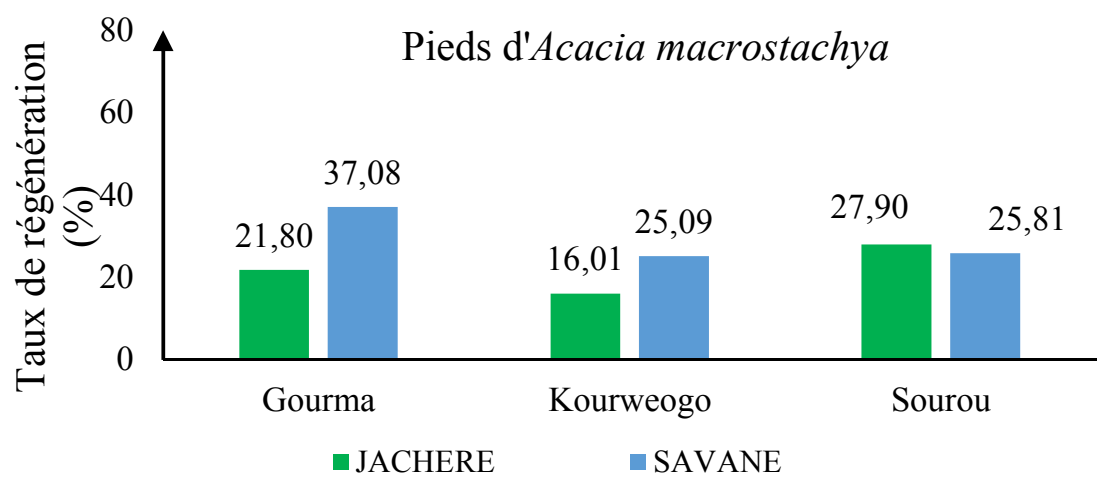

Figure 4: Régénération des individus des peuplements et des pieds de Senegalia macrostachya. 


\section{DISCUSSION}

Le nombre d'espèces recensées montre une flore riche avec au total 91 espèces ligneuses réparties en 55 genres et 28 familles. Cependant, ces résultats diffèrent peu de ceux de Mbayngone et al. (2008) qui ont recensé 92 espèces réparties dans 64 genres et 31 familles dans le secteur Nord soudanien où se situent les sites d'étude. Cette différence constatée entre les résultats de la présente étude et ceux de Mbayngone et al. (2008) peut s'expliquer par l'action anthropique et à l'avancée du désert qui entraîne la disparition de certaines espèces limitant ainsi la diversité floristique et par le fait que son site d'étude soit situé dans une aire protégée. Sur le plan de la représentativité des familles, les résultats de l'étude rejoignent ceux de Thiombiano (2005), Ouédraogo (2006), et Mbayngone et al. (2008) qui ont remarqué la dominance des Fabaceae-Mimosoïdeae et des Combretaceae dans le secteur Nord-soudanien chez les ligneux. Selon Thiombiano et al. (2005) et Abdourhamane et al. (2013), la dominance de la famille des Combretaceae est un signe de la pression de l'homme ou de la dégradation des conditions climatiques. Et selon Arbonnier (2000), Wittig et al. (2004) et Thiombiano et al. (2006), le secteur Nord-soudanien est la zone de prédilection de plusieurs espèces de la famille des Fabaceae-Mimosoïdeae et des Combretaceae. Savadogo et al. (2007) et Mbayngone et al. (2008) ont cité la famille des Combretaceae comme la famille dominante des ligneux au Burkina Faso.

La famille des Fabaceae-Mimosoïdeae regroupe 50 genres et environ 3000 espèces. Les espèces de cette famille sont des arbres ou arbustes et rarement des herbacées, généralement spinescents. Senegalia macrostachya est un arbuste sarmenteux dont les tiges flexibles s'accrochent aux branches des arbres voisins et s'élèvent volontiers (Arbonnier, 2000). L'espèce s'épanouit plus dans les jachères que dans les savanes (Eyog Matig et al., 2002). Cependant, l'étude n'a pas montré de différence significative entre la densité des pieds de Senegalia macrostachya dans les jachères et les savanes des différents sites. Cela peut s'expliquer par le fait que les jachères soient à une phase de reconstitution avancée, avec une grande similarité floristique ligneuse que les savanes.

Les populations de l'espèce dans les jachères et les savanes des différents sites, présentent une forte représentativité des individus de petits diamètres et de petites tailles. Cela se justifie par la forme en «L» des histogrammes de distributions horizontale et verticale et la décroissance des courbes de tendance exponentielle. La dominance des individus de petits diamètres et de petites tailles pourrait s'expliquer par le fait que l'espèce soit un arbuste mais aussi par les mauvaises conditions climatiques (irrégularité des pluies et baisse de la pluviométrie) et la pression anthropique (coupes abusives de bois, feux de brousse) ne favorisant pas une bonne croissance des espèces (Bellefontaine et al., 2000). Associées à ces histogrammes, les courbes de tendance exponentielle expriment théoriquement la dynamique des individus de l'espèce, le coefficient de détermination $\mathrm{R}^{2}$ renseigne sur la relation qui existe entre les facteurs de perturbations externes et les pieds de l'espèce. Les valeurs du coefficient de détermination $\mathrm{R}^{2} \geq 0,5\left(\mathrm{R}^{2}=0,81 ; \mathrm{R}^{2}=0,80\right.$; $\left.R^{2}=0,83\right)$ et $\left(R^{2}=0,83 ; R^{2}=0,65 ; R^{2}=0,72\right)$ respectivement dans les jachères et les savanes, indique que les facteurs de perturbation externe (broutage, coupes, feux) n'ont pas d'influence sur les diamètres et les hauteurs des individus (Thiombiano, 2005). La diminution du nombre d'individus d'une classe à une autre indique une faible croissance des individus des classes inférieures vers les classes supérieures. Ces résultats sur la structure horizontale et verticale de l'espèce des différents sites présentent des peuplements stables, capables de recruter des individus dans le temps avec une évolution progressive, et ils concordent avec ceux obtenus par Adjonou et al. (2009), Abdourhamane et al. (2013), Kaboré et al. (2013) et Sanon et al. (2015), sauf dans la province du Sourou où la population de Senegalia macrostachya est en état de déséquilibre sur le plan verticale avec des 
valeurs du coefficient de détermination $\mathrm{R}^{2}<$ $0,50\left(\mathrm{R}^{2}=0,12\right.$ et $\left.\mathrm{R}^{2}=0,10\right)$. Ce déséquilibre serait liée à la pression que l'espèce subie (récolte intégrale des fruits murs, récolte avant maturité totale, feux de brousse, etc.) qui conduit à une mauvaise régénération de l'espèce et constitue une menace à la survie de cette dernière.

L'évaluation $\mathrm{du}$ potentiel de régénération naturelle dans les différents sites montre que le taux de régénération de Senegalia macrostachya est faible aussi bien dans les jachères que dans les savanes, avec de faibles potentiels de renouvellement. Ceci traduit une mauvaise régénération de l'espèce. Cette mauvaise régénération peut s'expliquer par le fait que Senegalia macrostachya est une espèce agroforestière (Eyog Matig et al., 2002), donc régénère peu dans les savanes de même lorsque les jachères vieillissent. Cela s'explique aussi par le fait que les jeunes plants sont très appétés par les animaux et sont vulnérables aux facteurs de dégradation, telles que la sécheresse, les feux selon Ouédraogo (2006) et Ousseina et al. (2015). Selon Taïta (2003), les espèces dont les fruits et/ou les graines sont consommés rencontrent généralement des problèmes de régénération. C'est le cas de Senegalia macrostachya dont les graines et les fruits sont consommés et prisés par les populations (Eyog Matig et al., 2002). Selon Thiombiano et al. (2010), le fait que les jeunes pieds de certaines espèces se regroupent en touffe ne favorise pas la croissance des individus de ces espèces car la compétition y est rude. C'est le cas de Senegalia macrostachya. Ces résultats témoignent des potentialités de l'espèce à procurer de la nourriture et des revenus par la vente des graines aux communautés locales. Mais de grands efforts de gestion des ressources naturelles ligneuses devraient être entamés pour leur conservation dans les champs de culture et les jachères. A cet effet, la sélection et la conservation des pieds de l'espèce Senegalia macrostachya devraient être associées aux activités de production des populations rurales.

\section{Conclusion}

La présente étude montre que la flore ligneuse des savanes et des jachères des provinces du Sourou, du Kourweogo et du Gourma est riche et diversifiée. Elle a révélé que les Combretaceae et les FabaceaeMimosoïdeae sont les familles dominantes dans le secteur Nord soudanien. Senegalia macrostachya a un bon potentiel en peuplements naturels en termes de densité dans les jachères et les savanes au niveau des trois sites du secteur Nord soudanien du Burkina Faso. Ces formations végétales renferment donc un bon potentiel de Senegalia macrostachya qui peut procurer de la nourriture, des remèdes et des revenus aux populations locales à travers une exploitation rationnelle.

Les populations de Senegalia macrostachya sont stables et équilibrés au niveau de la structure horizontale. Par contre elles sont déséquilibrées du point de vue vertical dans la province du Sourou du fait de la pression exercée sur l'espèce. Ces résultats confirme l'hypothèse émise qui dit que «Senegalia macrostachya possède un potentiel important dans les différents types de formations végétales et que son état de peuplement est stable et équilibré». Cependant, le taux de régénération de l'espèce est faible au niveau des deux formations végétales dans tous les sites. Ce faible taux de régénération de l'espèce pourrait limiter la disponibilité de cette espèce ligneuse alimentaire à long terme. Il faudra entreprendre des actions visant à: conserver le potentiel disponible de l'espèce; promouvoir la protection des jeunes plants à travers la sensibilisation des populations à une exploitation judicieuse de l'espèce; appliquer la RNA aux plantules et aux rejets de souche de l'espèce pour les permettre d'atteindre le stade adulte; produire les plants de Senegalia macrostachya en pépinière afin de procéder à des reboisements de l'espèce; une mise en place de verger à Senegalia macrostachya. 


\section{CONFLIT D'INTERETS}

Les auteurs déclarent qu'il n'y a aucun conflit d'intérêts.

\section{CONTRIBUTIONS DES AUTEURS}

Dans la réalisation de la présente étude, YS et SG ont élaboré le protocole de recherche, dirigé et encadré cette étude dans la collecte et traitement des données, ils ont aussi rédigé le manuscrit; ET a apporté son soutien dans le traitement des données et a participé à la rédaction du manuscrit; ANS a participé à la validation du protocole et à la relecture du document.

\section{REMERCIEMENTS}

Notre reconnaissance va aux producteurs et aux formatrices endogènes des communes de Tougan, Boussé, Laye et Tibga pour leur participation aux travaux de terrain.

\section{REFERENCES}

Abdourhamane H, Morou B, Rabiou H, Mahamane A. 2013. Caractéristiques floristiques, diversité et structure de la végétation ligneuse dans le Centre-Sud du Niger : cas du complexe des forêts classées de Dan kada Dodo-Dan Gado. Int. J. Biol. Chem. Sci., 7(3): 1048-1068. DOI: http://dx.doi.org/10.4314/ijbcs. v7i3.13

Adjonou K, Bellefontaine R, Kokou K. 2009. Les forêts claires du Parc national OtiKe'ran au Nord-Togo : structure, dynamique et impacts des modifications climatiques récentes. Sécheresse, 20(1e): e1-e10. DOI: $10.1684 / \mathrm{sec} .2009 .0217$

Arbonnier M. 2000. Arbres, arbustes et lianes des zones sèches d'Afrique de l'Ouest. CIRAD, MNHN, UICN, 541p.

Bellefontaine R, Gaston A, Petrucci Y. 2000. Management of Natural Forest of Dry Tropical Zones. Conservation Guide, $n^{\circ} 32$. Food and Agricultural Organization (FAO). Rome.

Bognounou F. 2009. Restauration écologique et gradient latitudinal : utilisation, diversité et régénération de cinq espèces de Combretaceae au Burkina Faso.
Thèse de doctorat, Université de Ouagadougou, Unité de recherche et de formation en Sciences de la Vie et de la Terre, Burkina Faso, 179 p.

Dipama JM. 2010. Principaux facteurs environnementaux du Burkina Faso. In Atlas de la Biodiversité du Burkina Faso: le Milieu Biophysique, Thiombiano, K (eds). University Press: Franckfurt; 122-134.

Eyog Matig O, Gaoue OG, Dossou B. 2002. Réseau " Espèces Ligneuses Alimentaires ». Compte rendu de la première réunion du Réseau tenue 11-13 décembre 2000 au CNSF Ouagadougou, Burkina Faso. Institut International des Ressources Phytogénétiques. IPGRI Regional Office for sub-Saharan Africa c/o ICRAF: Nairobi, Kenya.

Ganaba S. 2011. La végétation Ligneuse du Sahel (Burkina Faso). Caractérisation, Utilisations, Tests de Restauration et Gestion. Editions Universitaires Européennes, 294p,

Kaboré E, Sambaré O, Ouédraogo A, Thiombiano A. 2013. Diversité et structure des cordons ripicoles le long de la sirba (Nord-Est du Burkina Faso). Int. J. Biol. Chem. Sci., 7(5): 1929-1950. DOI: http://dx.doi.org/10.4314/ijbcs. v7i5.13

Mbayngone E, Thiombiano A, Hadjali KH, Guinko S. 2008. Caractéristiques écologiques de la végétation ligneuse du Sud-Est du Burkina Faso (Afrique de l'Ouest): le cas de la réserve de Pama. Candollea 63: 17-33.

MECV (Ministère de l'Environnement et du Cadre de Vie). 2004. Audit environnemental de l'Autorité de Mise en Valeur de la Vallée du Sourou. 89p.

Mouamfon M, Guedje NM, Pepainyiene I, Zapfack L, Ngueguim JR, Lejoly J. 2015. Pentaclethra macrophylla Benth dans la forêt communautaire de Payo (Est-Cameroun): inventaire, productivité et commercialisation. Int. J. Biol. Chem. Sci., 900-216.DOI: http://dx.doi.org/10.4314/ijbcs.v9i1.18 
Ouédraogo A. 2006. Diversité et dynamique de la végétation ligneuse de la partie orientale du Burkina Faso. Thèse de doctorat de l'Université de Ouagadougou, p.195.

Ousseina S, Fortina R, Hamani Marichatou H, Yenikoye A. 2015. Diversité, structure et régénération de la végétation ligneuse de la Station Sahélienne Expérimentale de Toukounous, Niger. Int. J. Biol. Chem. Sci., 9(2): 910-926. DOI: http://dx.doi.org/10.4314/ijbcs.v9i2.29

Sanon Z, Hien M, Yameogo JT, Bachmann Y, Somda I. 2015. Dynamique structurale des îlots de forêt claire à Anogeissus leiocarpa (DC.) Guill. Et Perr dans le Sud-Ouest du Burkina Faso. Int. J. Biol. Chem. Sci., 9(2): 847-860. DOI: http://dx.doi.org/10.4314/ijbcs.v9i2.24

Savadogo P, Tigabu M, Sawadogo L, Oden PC. 2007. Woody species composition, structure and diversity of vegetation patches of a Sudanian savanna in Burkina Faso. Bois et Forêts des Tropiques, 294(4): 5-20.

Soloviev P, Zerbo GC, Lompo D, Yoda LB, Jacques D, Diallo A. 2009. Acacia Senegal au Burkina Faso : état de la ressource et potentiel productif. Bois et Forêts des Tropiques, 300(2)

Taïta P. 2003. Use of Woody Plants by Locals in Mare aux Hyppopotames, Biosphère Reserve in Western Burkina Faso. Biodiversity and Conservation. Academic Publishers: Kluwer; 1205 1217.

Thiombiano A. 2005. Les Combretaceae du Burkina Faso: taxonomie, écologie, dynamique et régénération des espèces. Thèse Doct. Etat, Univ. Ouagadougou. UFR/SVT 236p.

Thiombiano A, Schmidt M, Kreft H, Guinko S. 2006. Influence du gradient climatique sur la distribution des espèces de
Combretaceae au Burkina Faso (Afrique de l'Ouest). Candellea, 61: 189-213.

Thiombiano DNE., Lamien N, Dibong SD, Boussim IJ. 2010. Etat des peuplements des espèces ligneuses de soudure des communes rurales de Pobé-Mengao et de Nobéré (Burkina Faso). Journal of Animal \& Plant Sciences, 9(1): 11041116. DOI: http://www.biosciences. elewa.org/JAPS

Thiombiano DNE, Nieyidouba L, Dibong DS, Boussim IJ, Belem B. 2012. Le rôle des espèces ligneuses dans la gestion de la soudure alimentaire au Burkina Faso. Sécheresse, 23: 86-93.

Tindano E, Ganaba S, Thiombiano A. 2011. Rocky Woody Vegetation Diversity and Structure in the Oursi Dam Area, Northern Burkina Faso. ISESCO Journal of Science and Technology, 7(12): 15 28.

Tindano E, Ganaba S, Thiombiano A. 2014. Composition floristique et état des peuplements ligneux des inselbergs suivant un gradient climatique au Burkina Faso (Afrique de l'Ouest). Flora et Vegetatio Sudano-Sambesica, 17: 9$27 . \quad$ DOI: http://nbnresolving.de/urn/resolver.

Traore L, Ouedraogo I, Ouedraogo A, Thiombiano A. 2011. Perceptions, usages et vulnérabilité des ressources végétales ligneuses dans le Sud-Ouest du Burkina Faso. Int. J. Biol. Chem. Sci., 5(1): 258-278. DOI: http://ajol.info/index.php/ijbcs

Wittig R, Schmidt M, Thiombiano A. 2004. Cartes de distribution des espèces du genre Acacia L. au Burkina Faso. Etudes flor. vég. Burkina Faso 8: 19-26. 